\title{
Single-Crystal Growth of $f$-Electron Intermetallics in a Tetra-Arc Czochralski Furnace
}

\author{
M. SZlaWSKA* AND D. KACZOROWSKI \\ Institute of Low Temperature and Structure Research, Polish Academy of Sciences \\ P Nr 1410, 50-950 Wrocław, Poland
}

\begin{abstract}
The Czochralski technique has induced technological revolution in the electronic industry. It has also been widely used in the fundamental research area, as it allows obtaining high-quality single crystals of large variety of binary, ternary and multinary compounds. The unique tetra-arc Czochralski furnace installed at the Institute of Low Temperature and Structure Research, Polish Academy of Sciences in Wrocław, described in detail in the article, has been used to prepare a large number of single crystals of various Ce- and U-based intermetallic phases. Their excellent quality has facilitated advanced investigations of their anisotropic, often highly unusual physical properties.
\end{abstract}

DOI: 10.12693 /APhysPolA.124.336

PACS: 06.60.Ei, 75.30.Gw

\section{Introduction}

The Czochralski technique of crystal growth, named after distinguished Polish chemist Jan Czochralski, who invented his method in 1916 while investigating the crystallization rates of metals, is extensively used in semiconductor industry mainly for the purpose of obtaining large single crystals of silicon with low density of defects. Application of the technique has induced technological revolution in electronics, as it became the foundation in manufacturing microprocessors. Owing to that, since semiconductor integrated circuits are relatively cheap and easily available, advanced electronic devices are nowadays in common usage.

As a well-developed method of crystal growth, the Czochralski process is also important and widely employed in the fundamental research area. It is essential to investigate new materials on excellent quality single crystals. Though growing them is often quite a difficult task, the precise knowledge on anisotropic properties of the systems studied is invaluable. The Czochralski technique allows obtaining single crystals of large variety of compounds like borides, carbides, oxides, halides, salts, or intermetallic phases.

Since its discovery, the Czochralski method has been vastly developed. Scientists must have handled a lot of technical problems, associated e.g. with its adaptation to various types of materials. At present, different variants of the Czochralski furnaces are in common use. Setups utilized in scientific laboratories differ from each other primarily as regards the approach to heating and melting of starting materials. High-frequency electric induction heaters or resistance heaters are most widespread. However, their fundamental disadvantage is the presence of

*corresponding author; e-mail: m.szlawska@int.pan.wroc.pl a hot crucible. Sometimes, it is difficult to avoid contamination of the melt with crucible material, especially if the grown material has a high melting point. Some efforts were made to overcome this problem $[1,2]$. In 1968, Reed and Pollard [3] designed a cold-hearth tri-arc-melting furnace. In their approach, polycrystalline sample was melted by three electric arcs (to ensure uniform heating), which were ignited between tungsten cathodes and the sample placed on a water-cooled copper anode. Since thermal contact between the hearth and the sample was poor, the sample was molten, while the anode remained cold.

The Czochralski technique has however some restrictions. Above all, one can obtain single crystals only of systems that melt congruently. Otherwise, the phase decomposes below the melting temperature and the pulled ingot has multi-phase composition. Moreover, all the reactant elements must have rather low vapor pressure at high enough temperatures, which guarantees their negligible evaporation during the growth process that usually lasts several hours. If not, chemical composition of the melt changes during the synthesis and no single phase material is obtained.

Until now, the Czochralski pulling furnaces employing electric arcs are not common. Only few dozen laboratories worldwide are equipped with such devices. In Europe, the first multi-arc furnace was built in 1982 by Menovsky and Franse at the University of Amsterdam [4]. The tetra-arc furnace installed at the Division of Magnetics of the Institute of Low Temperature and Structure Research, Polish Academy of Sciences in Wrocław remains to date the only such facility available in Poland. Since its delivery to Wrocław in 2006, it has been used to pull up a large number of single crystals of various intermetallic compounds, with a special focus on $f$-electron materials.

Extraordinary interest in lantanoid- and actinoid-based systems, continuously shown by scientific com- 
munity since a few decades, arises due to very broad variety of different physical properties, which are directly linked to the character of their electronic $4 f$ or $5 f$ shells. The observed magnetic behavior, for example, may change from well-localized one, typical for "normal" rare-earths, to strongly delocalized one, characteristic of early actinides. The particularly intriguing features are often encountered in intermetallics based on cerium, ytterbium or uranium, primarily due to dual character of their $f$-states, controlled by hybridization of these states with electrons from conduction bands. As a consequence, plenty of unusual phenomena can arise, such as valence fluctuations, superconductivity, quantum criticality, heavy-fermion behavior, different exotic shortand long-range orderings with multiple order parameters.

Advanced investigations of these spectacular features require well-defined single-crystalline specimens. It has been proven that one of the best methods of growing crystals of cerium- and uranium-based intermetallics, which typically melt at quite high temperatures, is the Czochralski pulling technique. Its rare specific implementation in an arc furnace is a subject of the present article.

\section{Tetra-arc furnace design}

The tetra-arc furnace installed at the Institute of Low Temperature and Structure Research is presented in Fig. 1. The furnace chamber (seen on the right-hand side) is made of stainless steel. Its several lids can be independently opened so that possible contamination by solid residues of the chamber interior may be easily cleaned up. The furnace is equipped with a water-cooled copper crucible that plays a role of anode, on which the starting material is laid and melted. Uniform distribution of the melt temperature is ensured by four arc-electrodes located in quarters as well as by speed-controlled rotation of the hearth. Each electrode is made of tungsten doped with thorium oxide to facilitate arc switching on and its stability during growth process. The crystal preparation must be performed in oxygen- and moisture-free atmosphere in order to avoid oxidation of extremely air-sensitive $f$-electron materials. Therefore, the furnace is equipped with a high-vacuum pump station, which consists of highly efficient rotary and turbo-molecular pumps. For the growing process the chamber is filled with argon gas with attested purity supplied through a copper-made pipe line. Additionally, in order to further improve the high-purity of inner atmosphere, eventual traces of oxygen gas inside the chamber can be gettered by melting a piece of metallic titanium during the entire process of crystal growing. To generate electric arcs, the furnace is powered up by five independently controlled 200 ampere DC TIG welding power sources (partly seen behind the furnace chamber): four generators used for arcs that melt the sample button and one for melting the getter. A seed shaft unit, placed above the chamber, allows to pull up crystals with controlled speed of upward

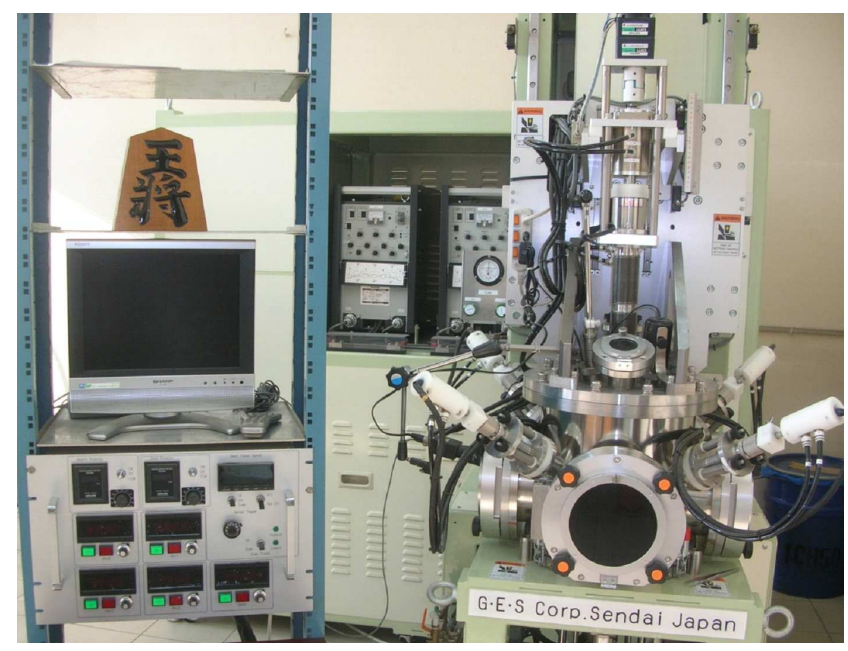

Fig. 1. The tetra-arc Czochralski furnace installed at the Institute of Low Temperature and Structure Research, Polish Academy of Sciences in Wrocław.

movement up to $39 \mathrm{~mm} / \mathrm{h}$ and controlled speed of axial rotation up to $10 \mathrm{rpm}$.

On the left to the furnace chamber (see Fig. 1), there is located the main control panel of the furnace, used for setting appropriate values of growth parameters, such as rate of pulling, speed of hearth and seed rotations, and intensity of electrical current engaged in the particular arcs. The pulling process can be observed through three side windows of the chamber, which are properly screened to protect eyes from intense ultraviolet radiation of the arcs. Moreover, the entire process is displayed on a TV monitor equipped with a CCD camera that looks inside the chamber.

\section{Crystal growth process}

To grow a single crystal of a given compound the crucible is filled with $5-7 \mathrm{~g}$ of starting material in the form of elemental constituents weighted in appropriate ratios. Alternatively, a pre-melted polycrystalline button with the proper composition can be used. If a seed crystal is available it is mounted on the bottom of substrate rod. Then, the chamber is evacuated to pressure of about $5 \times 10^{-6}$ Tr and filled with ultra-pure argon almost up to atmospheric pressure. Subsequently, the arcs are ignited one by one to melt first the getter and after a while the starting material. After some time needed to homogenize the hot melt, a sharpened tip of tungsten-made substrate rod is shortly dipped into it, and then pulled up quickly to form a neck in the so-nucleated crystal. This procedure is applied to avoid synthesizing polycrystalline material. In the next step, the diameter of the crystal being grown is gradually increased up to the required value by adjusting the pulling speed and lowering the melt temperature, realized through decreasing the intensities of arcs current. Optionally, the axial rotation of the substrate rod can be 
switched on and set at an optimal rate. The entire growing process takes several hours. The so-obtained single crystals have a shape of cylindrical rods with typical diameter up to 3-4 $\mathrm{mm}$ and length of a few dozen $\mathrm{mm}$. As an example, Fig. 2 displays on a few photographs the pulling process of a single crystal of $\mathrm{CeRh}_{3} \mathrm{Si}_{2}$, which was obtained from polycrystalline button. This particular crystal was then verified by standard characterization methods for its very high quality and thereafter used for a series of physical measurements [5-7].
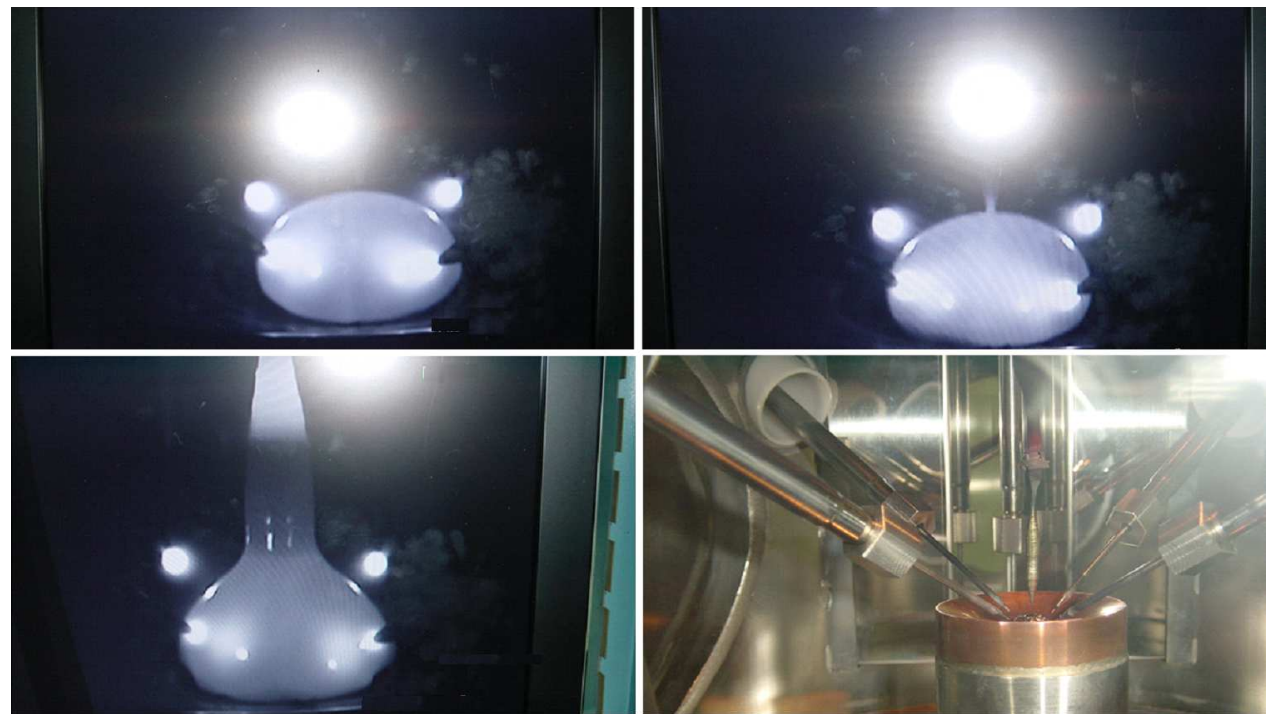

Fig. 2. Key stages of single-crystal growth process: (a) melting polycrystalline button, (b) seeding a crystal, (c) pulling up, (d) as-grown single crystal inside the chamber just after switching off the arcs.

\section{Properties of single crystals}

As an example of numerous f-electron based intermetallics obtained so far in the Crystal Growth Laboratory of the Institute of Low Temperature and Structure Research in the form of single crystals, Fig. 3 displays a Czochralski grown crystal of the ternary compound $\mathrm{UNiSi}_{2}$. High quality of the obtained crystal was inferred from the observation of well-defined sharp spots in the X-ray diffraction pattern recorded on a four-circle diffractometer, and corroborated in the electrical resistivity measurements via large values of the residual resistivity ratio. The compound was initially studied on polycrystalline samples and characterized as a ferromagnetic $\left(T_{\mathrm{C}}=95 \mathrm{~K}\right)$ Kondo lattice [8]. Its orthorhombic unit cell of the $\mathrm{CeNiSi}_{2}$-type (space group $\mathrm{Cmcm}$ ) was found strongly elongated along one of the principal crystallographic directions $(a=402.8 \mathrm{pm}, b=1614.1 \mathrm{pm}$, $c=402.7 \mathrm{pm}$ ), and hence pronounced anisotropy in the magnetic and transport properties could be anticipated. However, only the most recent reinvestigation carried out on oriented single crystals of $\mathrm{UNiSi}_{2}$ has provided an unambiguous proof for extremely anisotropic behavior of this compound.

As can be inferred from Fig. 4, in the ferromagnetically ordered state, the magnetization measured along the $c$-axis, $\sigma_{c}$, is distinctly larger than that taken along the $b$ - and $a$-axes, $\sigma_{b}$ and $\sigma_{a}$, respectively. The huge magnetic anisotropy is reflected in the ratios $\sigma_{c} / \sigma_{b} \approx 50$

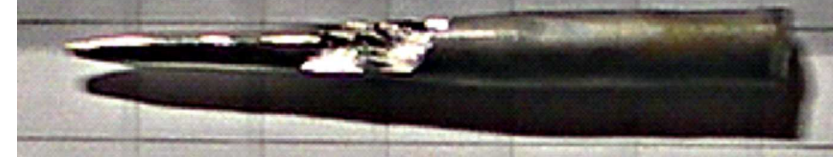

Fig. 3. Single crystal of $\mathrm{CeRh}_{3} \mathrm{Si}_{2}$ grown by the Czochralski technique in the tetra-arc furnace installed in Wrocław.

and $\sigma_{c} / \sigma_{a} \approx 4$, observed at $T=1.71 \mathrm{~K}$ in a magnetic field of $50 \mathrm{kOe}$. Clearly, the crystallographic $c$-axis is the easy magnetization direction, while the $b$-axis is the hard magnetic direction in the compound studied.

Figure 5 demonstrates that the magnetic behavior in $\mathrm{UNiSi}_{2}$ is strongly anisotropic also in the paramagnetic region. Both the magnitude and the temperature variation of the reciprocal magnetic susceptibility measured along the $b$-axis are distinctly different from those observed along the two other principal directions. Fitting the Curie-Weiss law to the experimental data over extended temperature intervals yielded the effective magnetic moment of $3.73 \mu_{\mathrm{B}}$ for the $b$-axis, while it is equal to $2.55 \mu_{\mathrm{B}}$ and $2.49 \mu_{\mathrm{B}}$ for the $a$-axis and the $c$-axis, respectively. In turn, the paramagnetic Curie temperature, derived from the $b$-axis data is negative and as large as $-417 \mathrm{~K}$, whereas it is positive and amounts to $2 \mathrm{~K}$ and $102 \mathrm{~K}$ for the $a$-axis and the $c$-axis, respectively. All these findings can be satisfactorily accounted for in terms of 


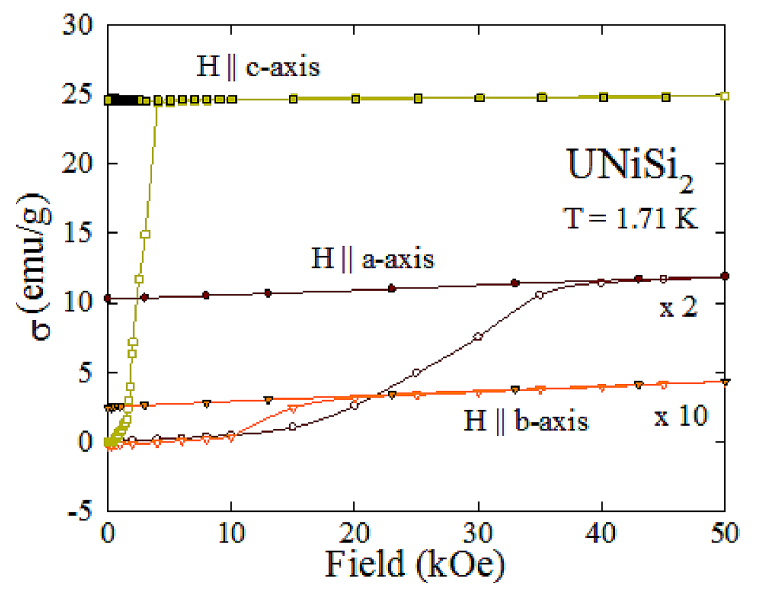

Fig. 4. Magnetic field variations of the magnetization of single-crystalline $\mathrm{UNiSi}_{2}$ measured in the ferromagnetically ordered region at the temperature of $1.71 \mathrm{~K}$. For the sake of clarity, the magnetization data obtained for the $a$ - and $b$-axes were multiplied by a factor of 2 and 10 , respectively.

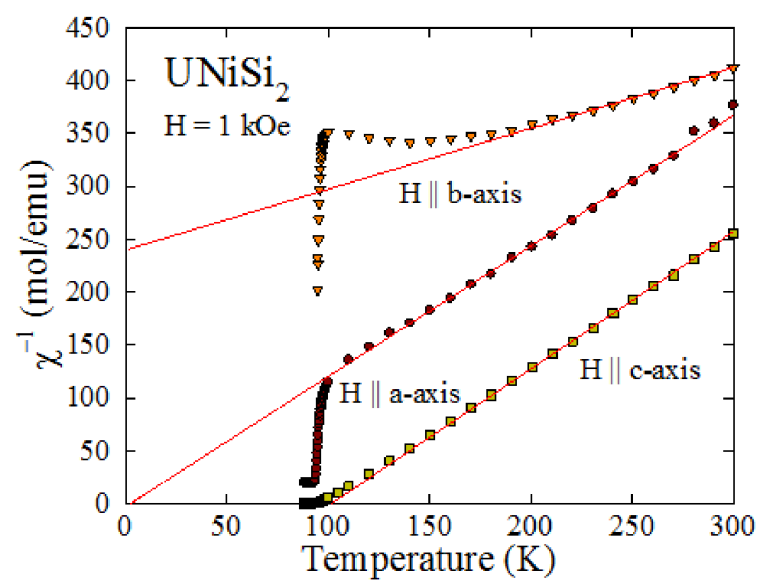

Fig. 5. Temperature dependences of the reciprocal molar magnetic susceptibility of single-crystalline $\mathrm{UNiSi}_{2}$ measured in the paramagnetic region in an applied magnetic field of $1 \mathrm{kOe}$. The solid straight lines represent the Curie-Weiss fits.

substantial single-ion magnetocrystalline anisotropy that is associated with fairly well localized $5 f$-electrons [9].

\section{Concluding general remarks}

Comprehensive investigations of well-defined single crystals are essential in contemporary solid state physics for both gaining fundamental knowledge on the physical properties of matter and recognizing their specific characteristics being prospective for practical applications. Frequently, experimental studies performed on single-crystalline materials provide facts and figures which cannot be inferred from analogous characterization carried out on polycrystals. Most straightforwardly, various physical property measurements executed along different crystallographic directions deliver information about anisotropic behavior of the studied material.

Since a few years, the tetra-arc Czochralski furnace installed at the Institute of Low Temperature and Structure Research, Polish Academy of Sciences in Wrocław, has been successfully employed to prepare numerous excellent-quality single crystals of various $f$-electron-based intermetallics. Unique implementation of four electric arcs allows growing materials with high melting temperatures. The obtained crystals can be used in detailed physical behavior studies, performed in multi-critical conditions: at low temperatures, in strong external magnetic fields and/or under high hydrostatic pressure. Continuous efforts directed towards preparation of brand new or previously poorly investigated systems, and methodical examining them on single-crystalline samples by means of a variety of advanced experimental techniques has been proven the best way to gain comprehensive knowledge about the materials important for modern science and technology.

\section{Acknowledgments}

The authors wish to express their gratitude to Professor Takemi Komatsubara for his kind personal involvement in the design and installation of the tetra-arc furnace at the premises of the Institute of Low Temperature and Structure Research, Polish Academy of Sciences in Wrocław, as well as his generous patience in teaching us how to prepare single crystals by the Czochralski technique and freely sharing with us his unique and vast experience in crystal growth methods.

\section{References}

[1] F.R. Monforte, F.W. Swanekamp, L.A. Van Uitert, J. Appl. Phys. 32, 959 (1961).

[2] R. Nitsche, J. Appl. Phys. 36, 2358 (1965).

[3] T.B. Reed, E.R. Pollard, J. Cryst. Growth 2, 243 (1968).

[4] A. Menovsky, J.J.M. Franse, J. Cryst. Growth 65, 286 (1983).

[5] D. Kaczorowski, T. Komatsubara, Physica B 403, 1362 (2008).

[6] A.P. Pikul, D. Kaczorowski, Acta Phys. Pol. A 115, 235 (2009).

[7] A.P. Pikul, D. Kaczorowski, Z. Gajek, J. StępieńDamm, A. Ślebarski, M. Werwiński, A. Szajek, Phys. Rev. B 81, 174408 (2010).

[8] D. Kaczorowski, Solid State Commun. 99, 949 (1996).

[9] D. Kaczorowski et al., to be published. 